\title{
Development of a Cockpit-Pilot Model for Thermal Comfort Optimization During Long- Mission Flight
}

Jörg Schminder, Roland Gårdhagen, Elias Nilsson, Karl Storck and Matts Karlsson

\section{Linköping University Post Print}

\section{Tweet}

N.B.: When citing this work, cite the original article.

Original Publication:

Jörg Schminder, Roland Gårdhagen, Elias Nilsson, Karl Storck and Matts Karlsson, Development of a Cockpit-Pilot Model for Thermal Comfort Optimization During LongMission Flight, 2016, AIAA Modeling and Simulation Technologies Conference San Diego, California, USA.

$\underline{\text { http://dx.doi.org/10.2514/6.2016-0172 }}$

Copyright: AIAA SciTech.

http://arc.aiaa.org/

Postprint available at: Linköping University Electronic Press

http://urn.kb.se/resolve?urn=urn:nbn:se:liu:diva-126538 


\title{
Development of a Cockpit-Pilot Model for Thermal Comfort Optimization During Long-Mission Flight
}

\author{
Jörg Schminder*, Roland Gårdhagen* and Elias Nilsson \\ Linköping University, Linköping, SE-581 83, Sweden \\ Karl Storck \\ SAAB AB, Linköping, SE-582 54, Sweden \\ and \\ Matts Karlsson* \\ Linköping University, Linköping, SE-581 83, Sweden
}

\begin{abstract}
The thermal comfort of a pilot is of crucial importance to maintain a high level of concentration and awareness during the entire flight mission. In this work a model for the thermal environment of the cockpit is developed and used as provider of input parameters to a thermoregulatory model, adopted from the literature, of a human. The cockpit-pilot model will be used to investigate and improve the thermal comfort for the pilot, particularly during longer flight missions.

In the cockpit model a combination of lumped systems and finite difference calculations is used to obtain input parameters, which are provided to the pilot model. The body, with clothes, is divided into 16 segments and a finite difference method is used to determine the temperature distribution within these. Several physiological mechanisms are included in the model.

Simulations with different boundary conditions show that the models work properly even for longer missions.
\end{abstract}

\section{Introduction}

Aircrafts are often exposed to a wide range of thermal conditions e.g. when operating at extremely hot airfields or when flying at high altitudes where temperatures are well below zero degrees Celsius. But also days with moderate outside temperatures (around $20^{\circ} \mathrm{C}$ ) can lead to a significant warming in the aircraft, especially in the cockpits of high performance aircrafts with large transparent canopies, when exposed directly to the sun over a longer period. The cockpit protects the pilot from this thermal life threat and provides a comfortable workspace during all circumstances.

Depending on the type of aircraft, the flight mission, and flight endurance thermal comfort can decline fast and significant heat stress is imposed on cockpit occupants especially when preparing for take-off or after landing with a limited or no climate control available. However, highest focus and error awareness are unconditional requirements for a pilot at any point in time while operating an aircraft. The thermal environment in the cockpit is a crucial factor when ensuring a high flight safety. ${ }^{1-3}$

Several potential models of the human body and heat transfer interaction with its surroundings have been presented, and are in general intended to be applicable for a range of applications. ${ }^{4-6}$

The study was initiated in order to increase the understanding for the thermal comfort in a cockpit for longendurance flights, how to model it, and in the long perspective to improve it. The aim of the present work

*Dept. of Management and Engineering. 
is to develop a model for the thermal environment in the cockpit, and combine it with the thermoregulatory model developed by Fiala..$^{7,8}$

\section{Materials and methods}

\section{A. The combined cockpit and pilot model}

The presented thermoregulatory model does not, like many other existing models, aim to perform for a wide range of different situations, but to a specific aviation related application like the yet complex thermal interaction between the cockpit and the pilot. For this reason a model was developed which combines the thermodynamics in a cockpit with a human thermoregulatory model representing the pilot. The cockpit model was developed for this study and combines lumped systems with finite difference modeling. It provides constantly updated input parameters, which can change significantly during a flight mission, to the pilot model.

The combined model consists of five sub-models, or parts: one for the ambiance in which the aircraft is operating, one for the cockpit interior, one for the cockpit hood, one for the cockpit air, and one for the pilot. The first four parts are simulated in the cockpit model whereas the pilot's thermal response is computed by the human thermoregulatory model. The different parts of the combined model are illustrated in Figure 1.

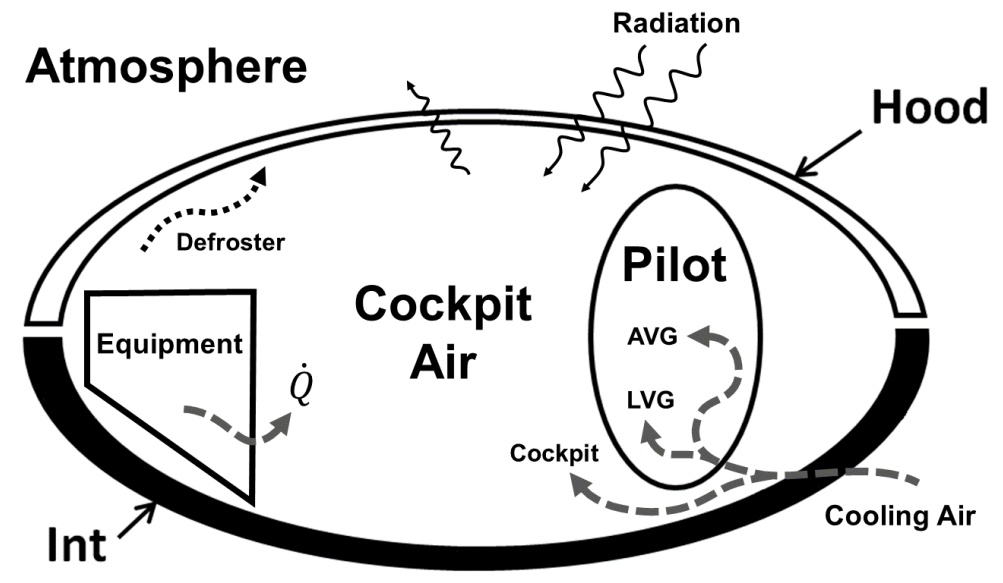

Figure 1. Schematic illustration of the combined cockpit-pilot model. The atmosphere, hood, interior, and air are parts of the cockpit model, which provides the pilot model with input parameters. There is also convective and radiative heat transfer inside the cockpit.

Between the different parts the basic heat exchange mechanisms convection, conduction, and radiation take place according to the following list.

- Convection

- Between the pilot and the cockpit air.

- Between the interior and the cockpit air.

- Between the hood and the cockpit air.

- Between the hood and the surroundings.

- Conduction

- Within the pilot.

- Within the hood.

- Radiation

- Between the interior and the hood. 
- Between the interior and the surroundings.

- Between the hood and the surroundings.

An assumption which was made is that the heat transfer within the interior and the cockpit air is sufficiently large so that these parts can be modeled as lumped systems.

\section{B. The Pilot Model}

To get a better understanding of how a pilot's body responds to various thermal cockpit conditions a pilot model based on Westin's improved thermoregulatory model, ${ }^{9}$ which in turn has Filas thermoregulatory model $^{7,8}$ as foundation, was implemented. The model was chosen because it is considered to be the most suitable to use for a pilot and because it has been validated for a broad amount of environments.

For the purpose of simulating the human body as close as possible the body is divided into 16 parts: head, neck, thorax, abdomen, upper arms, forearms, hands, thighs, calves, and feet. The head is the shape of a sphere and the rest of the body parts are shaped as cylinders. The geometrical properties of the body and parameters such as weight and basal metabolic rate are estimations based on a typical trained human. A sketch of the model is shown in Figure 2.

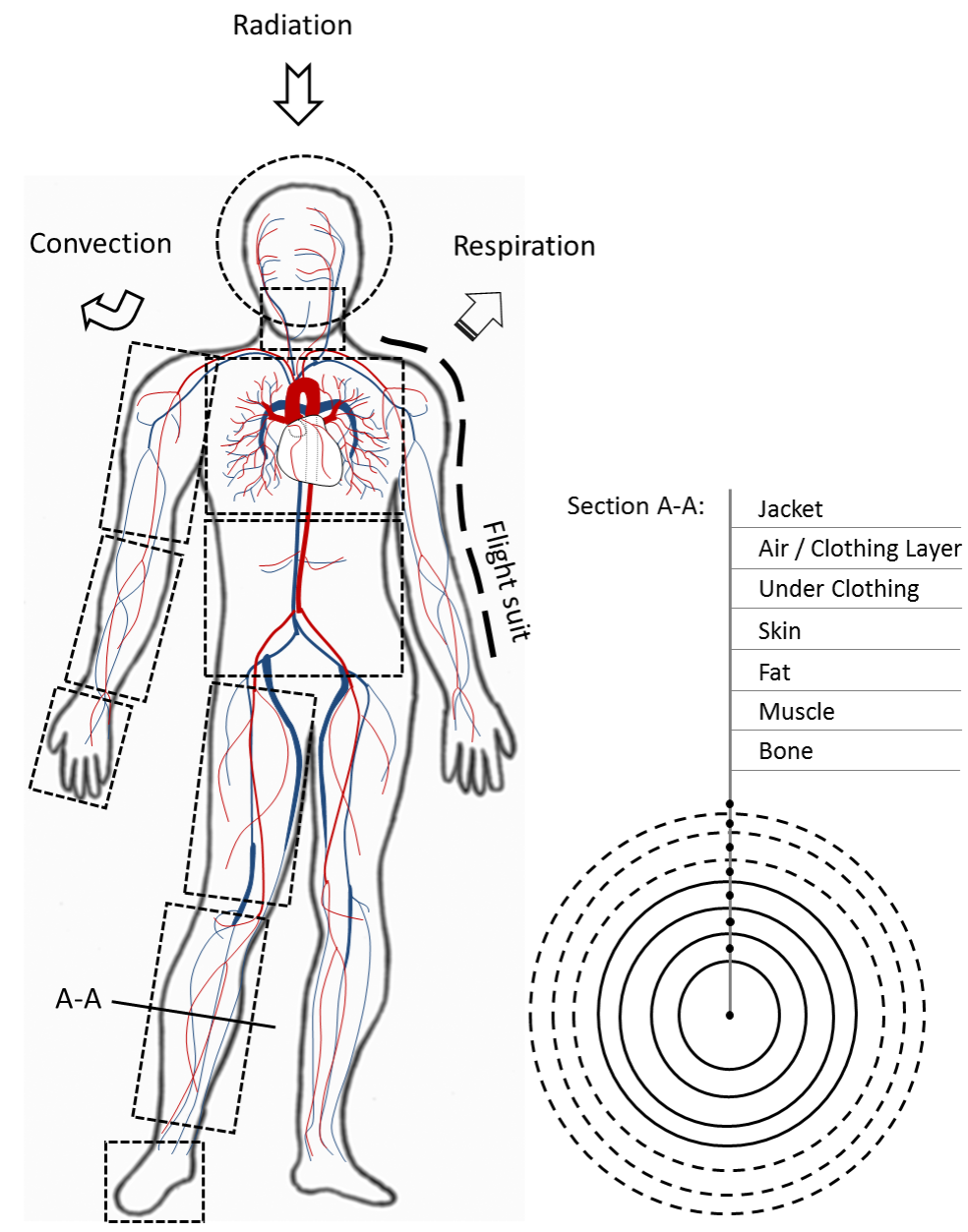

Figure 2. A schematic diagram of the parts of the pilot model and the pilot's passive thermoregulatory system.

The majority of the body parts have four layers: bone, muscle, fat, and skin, see Figure 2. The exceptions are the head, the thorax, and the abdomen. The head has an inner layer with the brain, the thorax contains the lungs as inner layer, and the abdomen has viscera as inner layer. The head, thorax, and abdomen then 
have bone, muscle, fat, and skin layers like the rest of the body parts. The amount of bones and tissues in each layer is handled by setting outer radii on the layers for each body part. Since the layers are made of different materials they have different properties. Parameters such as blood perfusion rate and skin sensitivity coefficient are different for each body part and are therefore set separately.

In addition to that pilots wear special clothing which depends on the aircraft type, mission, and environmental conditions. The pilot in this study has a clothing set-up implemented like that used by pilots flying in warm conditions. The clothes are divided into three layers. Closest to the skin there is a layer underclothing, socks, or hoods used under the helmet. In the middle there is a combined air and clothing layer to simulate the space between clothes, e.g. between under clothing and the jacket. On top there is a final layer to simulate the outer layer of clothes, e.g. the jacket, gloves, or shoes. The pilot model consists of two systems: the passive system which simulates the heat transport within the human body plus the heat exchange between the pilot and the environment (cockpit), and a control system which senses thermal changes in the state of the body and responds with shivering, sweating, vasodilation, vasoconstriction, and respiration.

The base for the passive model is Pennes' one dimensional bioheat equation ${ }^{10}$ for spherical and cylindrical geometries, presented in Equation 1, which describes the heat transport in each tissue layer.

$$
k\left(\frac{\partial^{2} T}{\partial r^{2}}+\frac{\omega}{r} \frac{\partial T}{\partial r}\right)+\beta\left(T_{b l, a}-T\right)+q_{m}=\rho c \frac{\partial T}{\partial t}
$$

where $\omega$ is set to 1 for cylindrical geometries and 2 for spherical geometries. $\beta$ is the blood perfusion rate factor, $r$ is the local node radius, and $q_{m}$ is the metabolic heat generation rate.

The implemented control system in this model is based on the commonly used Stolwijk-Hardy error function concept. The control system receives data about the current temperatures and then creates an error signal based on the difference between the current temperatures and temperatures at thermal neutrality in each node.

The bodys physiological responses to extreme high or low temperatures, which can be reached in high performance aircraft cockpits, might not be sufficient to maintain all life functions. External heating or cooling systems are needed to ensure basic thermal comfort so that the pilot can hold up a maximum in flying performance under these extreme thermal conditions. Beyond the ordinary cockpit conditioning system the model offers additional pilot cooling through an air ventilated garment (AVG) on the thorax and leg ventilated garment (LVG) on the thighs and calves. A simplification in this model is that air coming from the AVG and LVG only affects body parts directly underneath the ventilated garments and not diffuses so that other body parts are cooled as well.

\section{The Cockpit Model}

The pilot's comfort is influenced by different outside factors for example the solar irradiance, altitude, flight speed, or flow temperature from the air conditioning system. All these are directly or indirectly input parameters to the pilot model provided by the cockpit model.

As presented in Figure 1 the cockpit model consists of the surrounding of the aircraft, the hood, the interior, and the cockpit air. For each of these parts the model calculates the relevant heat transfer mechanisms (i.e radiation, convection, and conduction) between the different components.

\section{Atmosphere}

The variation of the properties in the surrounding of the aircraft like temperature and pressure at different altitudes are calculated by simulating the international standard atmosphere (ISA). However, due to aerodynamic heating the static air temperature (SAT) may rise depending on the flight speed and altitude. For this reason the model calculates the recovery air temperature to take the possible additional heating into account. 
Apart from the ambient temperature, over the day the sun is a major heat source into the cockpit. How much of the sun radiation that reaches the inside of the cockpit or is absorbed by the hood is determined by the models for hood and internals of the cockpit.

\section{The Hood}

To provide the pilot in high performance aircrafts with a good view during all maneuvers large transparent cockpit canopies are often installed. The cockpit model handles this hood as a translucent cover assuming the properties of polymethylmethacrylate. The total, inside, and outside area, the thickness, the length, and the projected area of the hood are customizable for any type of aircraft.

The hood model calculates, using a finite difference method, the heat transfer through the hood. Radiation and convection to both the inside and the outside surface of the hood are considered. Heat transfer coefficient, both inside and outside, are computed using semi-empirical relations taken from the literature.

\section{The Interior}

The interior represents all solid material mounted inside the cockpit like the instrument panel, the seat, and all electronic facilities. Heat which is produced, emitted, and absorbed by the interior will contribute to a significant warming of the cockpit. The determined interior temperature will then serve as an input parameter to compute the cockpit air temperature.

The heat balance for the interior, Equation 2, includes the heat generated by the equipment $\left(\dot{Q}_{\text {equip,int }}\right)$, the heat from convection $\left(\dot{Q}_{c o n v, i n t}\right)$, radiation to and out of the interior $\left(\dot{Q}_{\text {rad,in/out }}\right)$, and the air flow from the cooling of the interior $\left(\dot{Q}_{\text {cool }, \text { int }}\right)$.

$$
\dot{Q}_{\text {int }}=\dot{Q}_{c o n v, \text { int }}+\dot{Q}_{\text {rad }, \text { in }}-\dot{Q}_{\text {rad }, \text { out }}+\dot{Q}_{\text {equip }, \text { int }}-\dot{Q}_{\text {cool }, \text { int }}
$$

Using the calculated net heat and the first law of thermodynamics, the temperature of the interior can be determined.

\section{The Cockpit Air}

The cockpit air temperature is a significant measurement for the thermal comfort of the pilot and is for this reason a central input parameter to the pilot model.

The temperature of the cockpit air depends e.g. on the heat exchange with the inside of the hood and the surface of the interior, and from the cockpit cooling air mass-flow. The air flow into the cockpit consists of warm cockpit avionic cooling air, cool air from the cockpit conditioning system, and air from the pilot cooling system. Like in the real aircraft the model offers the possibility that the mass flow and temperature from the cockpit conditioning system can be altered by the pilot with a temperature controller, located inside the cockpit.

As indicated above the cockpit-conditioning air is split into two mass flows before entering the cockpit. The first flow enters the clothing of the pilot and the cooling garments while the second mass flow proceeds directly to the cockpit. The fraction of air entering into the cockpit and the cooling garments/clothes is decided by the pilot through a vault. After the air passed the cooling garments the air is flowing with an average temperature from the AVG and LVG into the cockpit.

Air may also come from the defroster system that is used to remove fog or ice from the hood. This function is normally only turned on when the fighter is descending from a high altitude to a lower altitude. The mass flow of air from the defroster depends on the current altitude but the temperature remains constant.

The heat balance for the cockpit air, Equation 3, contains convection $\left(\dot{Q}_{c o n v}\right)$ from the interior, pilot, and hood. In addition all cockpit air flows are included these are: cooling air to the cockpit and pilot $\left(\dot{Q}_{\text {cockpit }}\right.$, $\left.\dot{Q}_{\text {pilot }}\right)$ as well as warm air from the equipment $\left(\dot{Q}_{\text {equipment }}\right)$, the avionic $\left(\dot{Q}_{\text {avionic }}\right)$, and the defroster system $\left(\dot{Q}_{\text {defrost }}\right)$ if activated. 


$$
\dot{Q}_{c p}=\dot{Q}_{c o n v, i n t}+\dot{Q}_{c o n v, p i l o t}-\dot{Q}_{c o n v, h o o d}+\dot{Q}_{\text {equipment }}+\dot{Q}_{\text {avionic }}+\dot{Q}_{\text {defrost }}-\dot{Q}_{\text {pilot }}-\dot{Q}_{c o c k p i t}
$$

Using the calculated total heat flow to and from the cockpit air it is possible for the model to compute the cockpit air temperature $\left(\dot{Q}_{c p}\right)$.

\section{Results and Discussion}

Figure 3(a) and 3(b) show the results of a flight from ground (MSL) up to an altitude of $30000 \mathrm{~m}$ with a constant Mach number (Ma) of 0.9. The aim with this simulation was simply to show the correct function of the standard atmosphere model delivering basic input parameter like air temperature and pressure to the cockpit model.

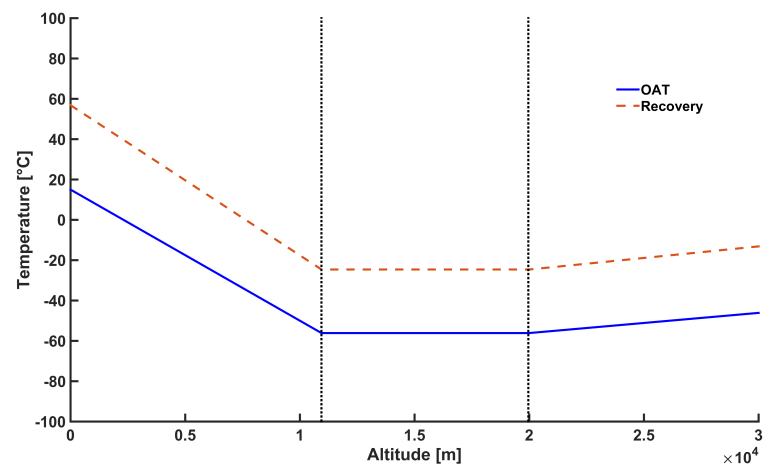

(a) Atmospheric and recovery temperature

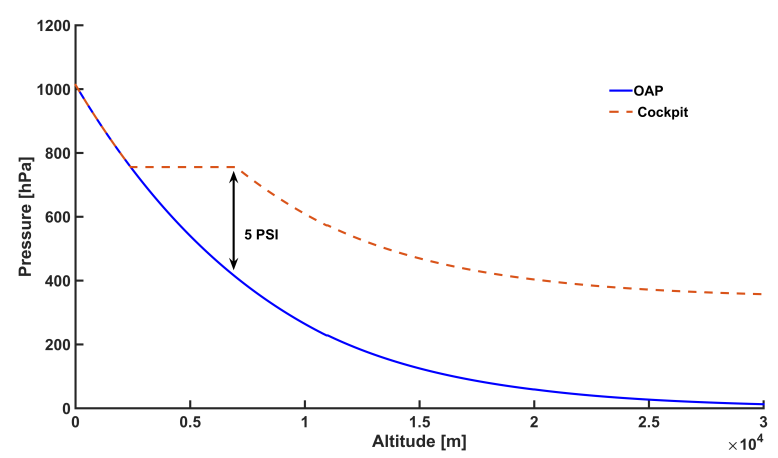

(b) Atmospheric and cockpit pressure

Figure 3. Outside air temperature and pressure from MSL up to $30000 \mathrm{~m}$ computed using the standard atmosphere. The stagnation temperature corresponds to Ma 0.9. The cockpit air pressure decreases with altitude until $2400 \mathrm{~m}(8000 \mathrm{ft})$, remains constant until a pressure difference of 5 psi is reached, and then maintains a constant difference of 5 psi.

For the outside air temperature the model shows, as expected, first a drop in temperature with a constant laps rate up to the end of the troposphere followed by an area where the temperature remains constant (tropopause) after which it starts to increase slightly after entering the stratosphere. In contrast to the outside air temperature the outside air pressure falls constantly with increasing altitude.

Apart from the above mentioned outside properties Figure 3(a) also shows the recovery temperature, which is higher due to the friction close to the outer surface of the aircraft. Figure 3(b) in turn includes the cockpit air pressure which drops in the same way as the outside air pressure until the aircraft reaches an altitude of 8000 feet where a pressure valve closes so that the cockpit pressure is constant until it reaches a difference of 5 psi compared to the outside pressure. After this the cockpit pressure starts to fall again in the same way as the outside air pressure but with an offset of 5 psi.

Next, a flight envelop starting directly with a take off from the ground (MSL) followed by a climb up to an altitude of $15000 \mathrm{~m}$ while increasing the Mach number from 0 to 0.9 was run. After reaching the cruising altitude, height and flight speed were maintained until the end, see figure 4 (a).

Figure 4 (b) shows the cockpit air temperature and the surface (i.e. inside and outside) temperatures of the hood. It is clear that the model is able to maintain a stable cockpit temperature despite changes like increased ground temperature (DTISA +10 ) and the decrease of outside air temperature when climbing to a high cruise altitude.

The temperature of the outside surface of the hood follows the recovery temperature closely, and the temperature offset caused by the higher ground temperature is kept during the entire flight. Figure 4 (c) presents 
the convective heat flows in the cockpit model. Figure 4 (d) presents the heat exchange due to different air flows in the cockpit together with the total heat exchange of the cockpit. It is apparent that the convective heat flow from the interior is dominating. It originates from the increased temperature difference between the interior and cockpit air, which in turn is due to the heating of the interior by sun radiation and the electronic equipment. The convective heat flow from the hood reaches a steady value after an initial balancing, and the pilots convective heat transfer to the cockpit is relatively low compared to the other convective heat flows.

To control and ensure a comfortable temperature for the pilot, cooling air is supplied to the cabin. The effectiveness of the cooling air system in the model is demonstrated by the 10 degree offset simulation. In addition to higher surrounding temperature the sun radiation was increased, thus causing considerable additional heating of the interior. Hence the cooling was increased, Figure 4 (d), which resulted in a relatively unchanged cockpit air temperature compared to standard day conditions. Despite the large changes in the separate heat flows the total heat flow stays steady to all time and tend to zero, thus indicating that the model works smoothly and that the different heat flows balance each other unless cooling or heating is required.

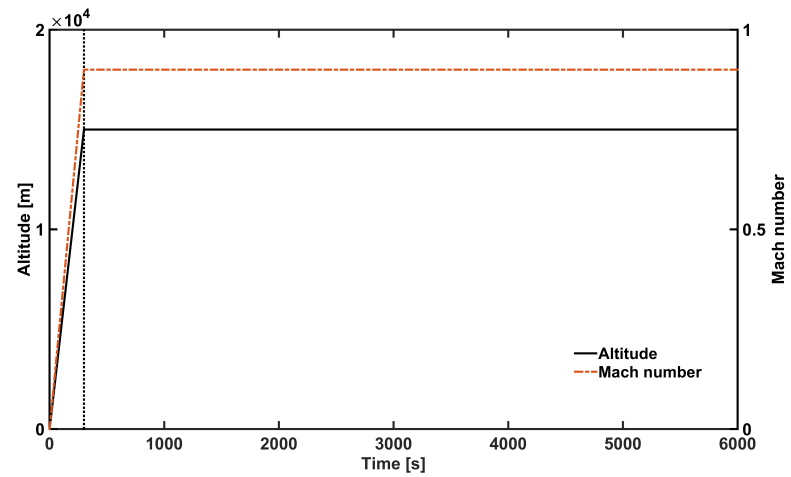

(a) Flight envelope

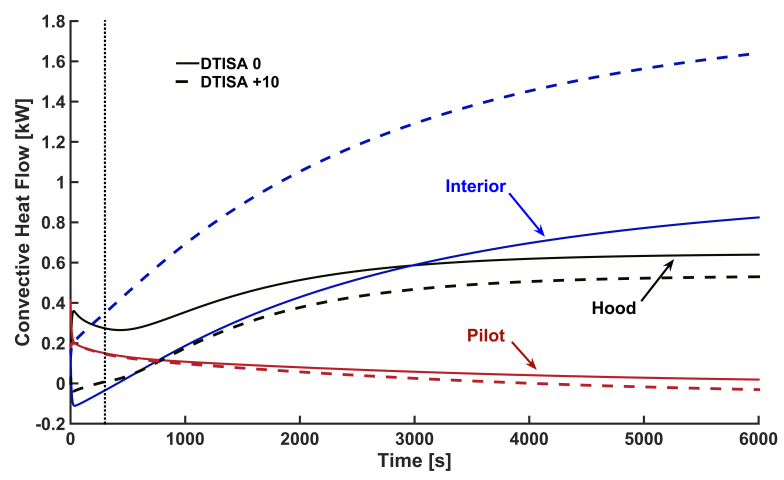

(c) Convective heat flow

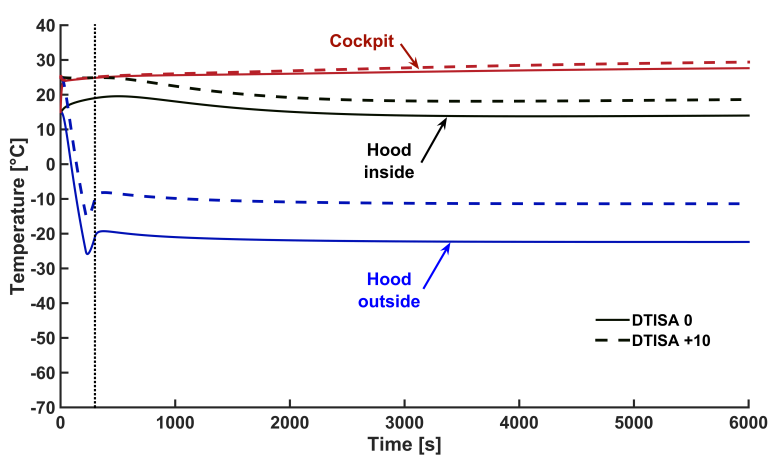

(b) Cockpit temperatures

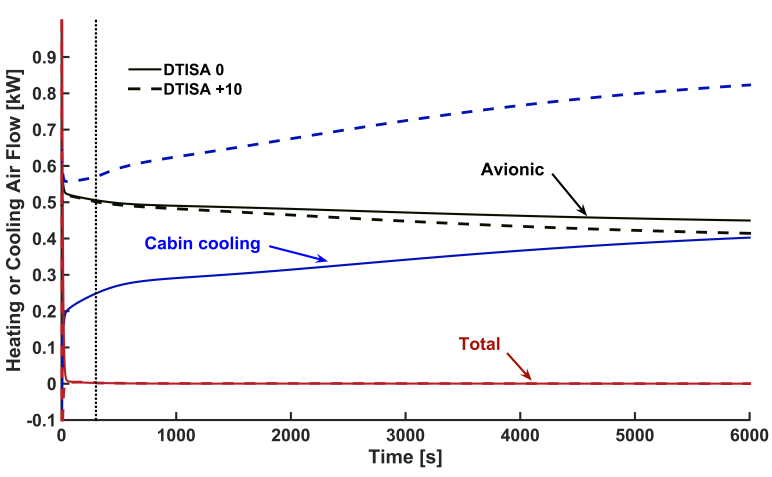

(d) Cockpit air flows

Figure 4. Details of the first flight case including cockpit temperatures and related heat flows.

The task of the cockpit model is to provide appropriate input data to the thermoregulatory model of the pilot. The temperatures of the cockpit air, the interior and the inside surface of the hood interact with the temperature of the surface of the pilot's cloths through convective and radiative heat transfer. Thus these temperatures affect the thermal comfort of the pilot and in turn the ability to successfully perform a complex and demanding mission after a long flight.

To demonstrate this interaction in flight a second envelop was created, Figure 5. It consists of a longer taxing on ground (MSL) followed by the take off including acceleration and a static climb to a first cruise altitude at $10000 \mathrm{~m}$. The mission then continued with a second climb to a higher altitude $(20000 \mathrm{~m})$ followed by a decent back to the first cruise altitude. After that the aircraft finally returned for landing including 
a constant decent and longer taxing at the destination airport. The flight case was tested for two different Mach numbers: Ma 0.5 and 0.9 .

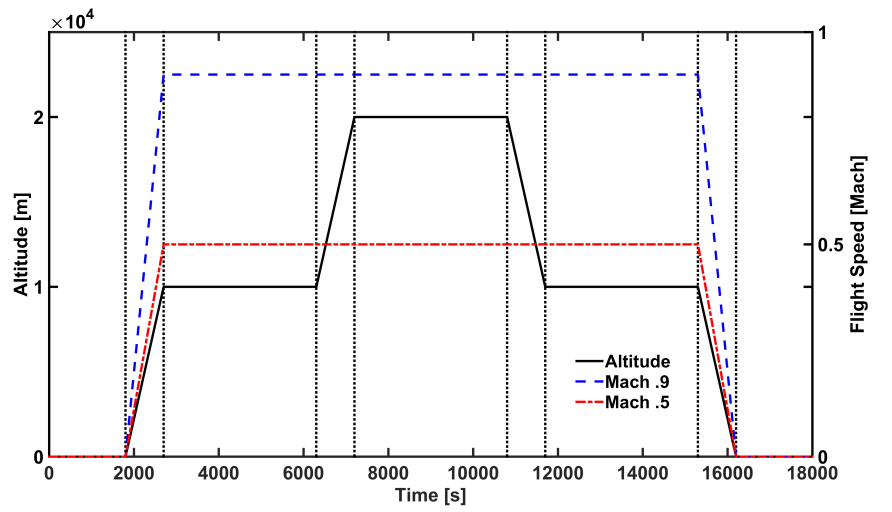

Figure 5. Flight envelope for the second case.

Figure 6 (a) shows that the cockpit related temperatures follow the atmospheric changes as expected. The temperature changes caused by the increase of Mach number are most distinct at the outside surface of the hood. The inside temperatures however remain relatively unaffected due to the insulation of the hood and cockpit air system. For these input parameters the results for the pilot model are presented in Figure 6 (b). While the outer surface temperature of the flight suit is low and responding to the changes of the surrounding temperatures the core and hypothalamus temperatures stay essentially unaffected. These results indicate that the thermoregulatory system of the pilot works properly.

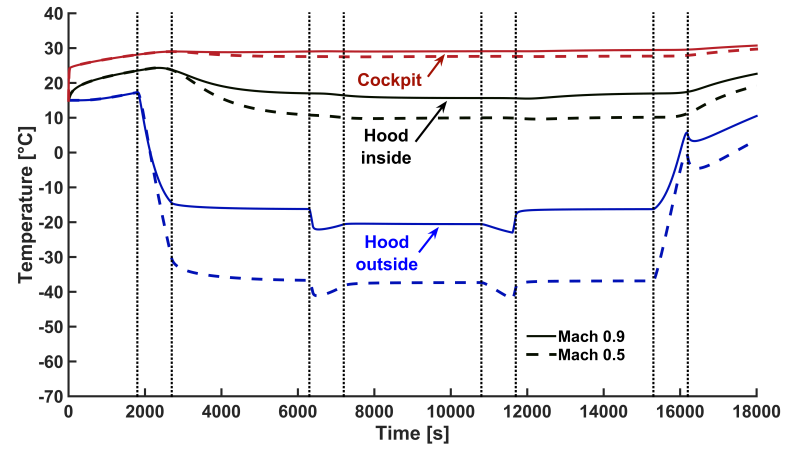

(a) Cockpit temperatures

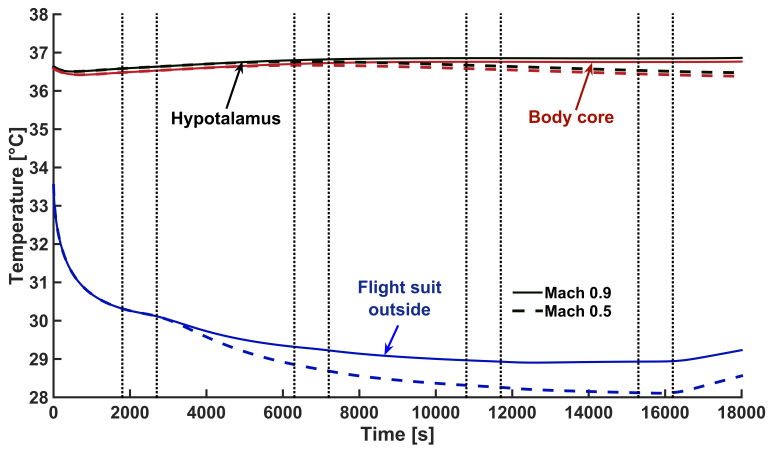

(b) Body temperatures

Figure 6. Cockpit and body temperatures during the second flight case together with energy transfer due to convective heat transfer and air flows in the cockpit.

Since a major aim with the project is to model thermal behavior of the cockpit and pilot under long flight missions the final flight envelop is a more realistic case imitating a ferry flight including aerial refueling after half of the ten-hour flight. The flight starts with a short taxing followed by climb to cruise altitude of $12000 \mathrm{~m}$ and thereafter flight at constant altitude. Five hours into the mission the aircraft descends to a lower altitude to meet the tanker aircraft after which the aircraft returns to its cruise altitude. The aircraft has now fuel for five more hours including descending and taxing at the destination airport.

The results for this case show that both the cockpit, Figure 7 (b), and the pilot model, Figure 7 (c), are able to hold a constant temperature over a long time. Furthermore it is shown that the model is able to return to its original values after a temporary deviation. The effect of the decent for refueling is most noticeable in the outside temperature of the hood. A minor reaction on the inside surface of the hood causes a moderate increase of convective heat flow from the hood, which though is damped out by the climate system so that the cockpit temperature remains unchanged. Consequently the pilot maintains his temperature during the 
entire flight.

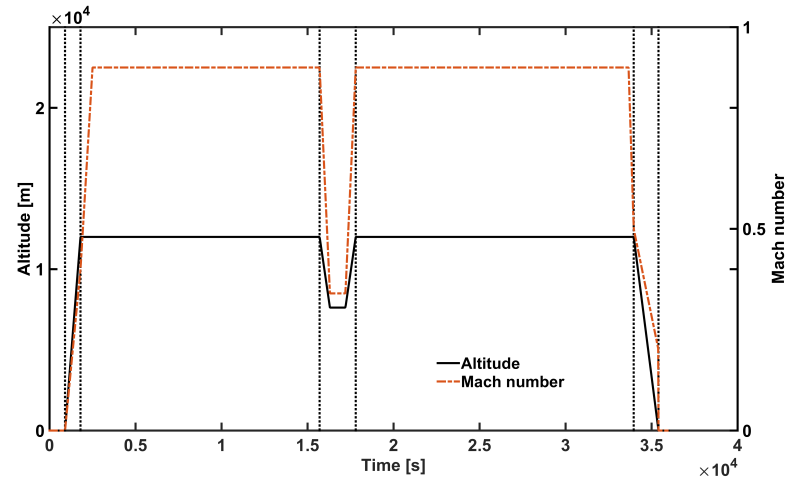

(a) Flight envelope

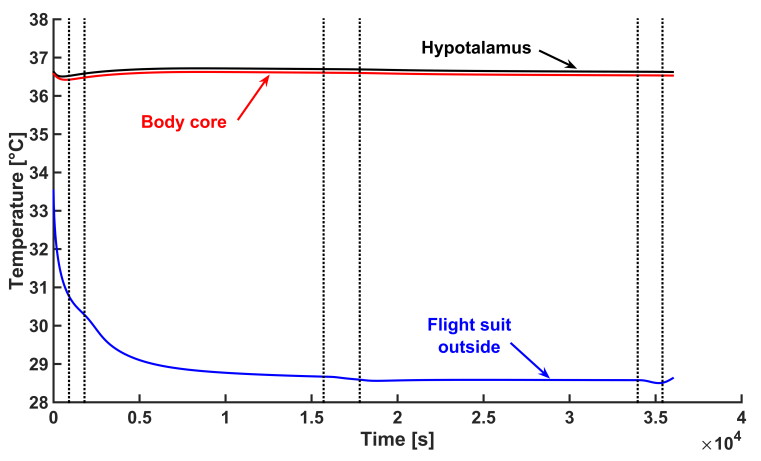

(c) Body temperatures

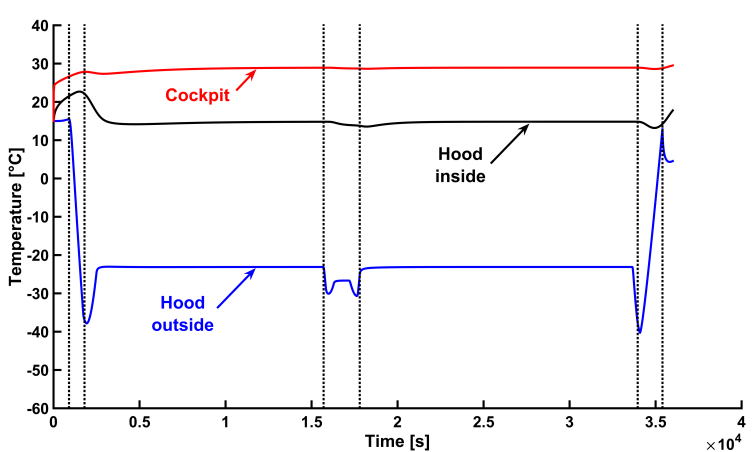

(b) Cockpit temperatures

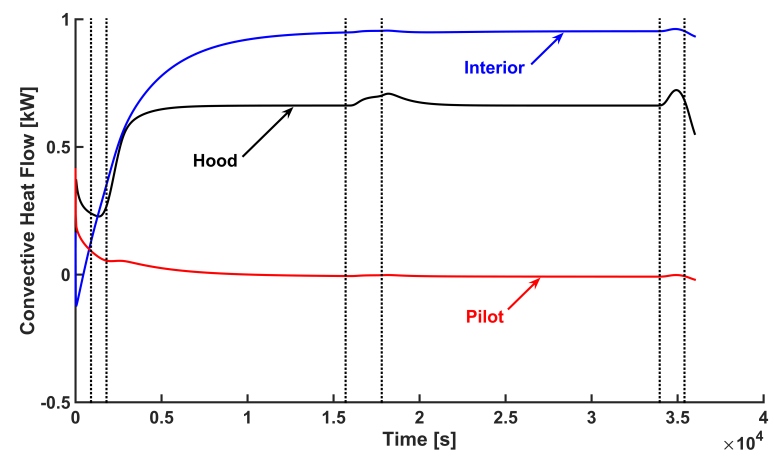

(d) Convective heat flow

Figure 7. Flight envelope, temperatures, and heat flows for the long mission flight case.

\section{Conclusion and Future Work}

It was shown that the cockpit-pilot model works properly for different flight cases. The combined model is suitable for pilot heat stress related studies and long range flight missions, which can be simulated within a reasonable time. Future work includes model validation as well as further development concerning the pilot model response to changes in the environment.

\section{References}

${ }^{1}$ S. A. Nunneley and C. F. Flick. Heat stress in the A-10 cockpit: Flights over dessert. Aviation, Space, and Enviromental Medicine, 52(9):513-516, 1981.

${ }^{2}$ S. A. Nunneley R. F. Stribley and J.R. Allan. Heat stress in front and rear cockpits of F-4 aircraft. Aviation, Space, and Enviromental Medicine, 52(5):287-290, 1981.

${ }^{3}$ S. A. Nunneley and L. G. Myhre. Physiological effects of solar heat load in a fighter cockpit. Aviation, Space, and Enviromental Medicine, 47(9):969-973, 1976.

${ }^{4}$ E.H.Wissler. A methematical model of the human thermal system. Bulletin of Mathematical Biophysics, 26:147-158, 1964.

${ }^{5}$ R.G.Gordon R.B. Roemer and S.M.Hrvath. Amethematical model of the human temperature regulatory system-transient cold exposure response. IEEE Transactions on Biomedical Engineering, 23(6):434-444, 1976.

${ }^{6}$ L.H.Kuznetz. A two-dimensional transient mathematical model of human thermoregulation. Journal of Applied Physiology, 237(5):266-277, 1979.

${ }^{7}$ D. Fiala K. J. Lomas and M. Stoher. A computer model of human thermoregulation for a wide range of enviromental conditions: the passive system. Journal of Applied Physiology, 87(5):1957-1972, 1999.

${ }^{8}$ D. Fiala K. J. Lomas and M. Stohe. Computer prediction of human thermoregulatory and temperature responses to a wide range of enviromental conditions. Journal of Applied Physiology, 45:143-159, 2001. 
${ }^{9}$ Johan K. Westin. An improved thermoregulatory model for cooling garment applications with transient metabolic rates. PhD thesis, University of Central Florida, Orlando, Florida, October 2008.

${ }^{10}$ H.H.Pennes. Analysis of tissue and aterial blood temperatures in the resting human forearm. Journal of Applied Physiology, 1(2):93-121, 11948. 